\title{
Delay-Dependent Robust Stabilization for Nonlinear Large Systems via Decentralized Fuzzy Control
}

\author{
Chun-xia Dou, ${ }^{1,2}$ Zhi-sheng Duan, ${ }^{1}$ Xing-bei Jia, ${ }^{2}$ Xiao-gang Li, $^{2}$ \\ Jin-zhao Yang, ${ }^{2}$ Ting Gui, ${ }^{2}$ and Ye-fei $\mathrm{Bi}^{2}$ \\ ${ }^{1}$ State Key Laboratory for Turbulence and Complex Systems, Department of Mechanics and \\ Aerospace Engineering, College of Engineering, Peking University, Beijing 100871, China \\ ${ }^{2}$ Institute of Electrical Engineering, Yanshan University, Qinhuangdao City 066004, China \\ Correspondence should be addressed to Zhi-sheng Duan, duanzs@pku.edu.cn
}

Received 14 October 2010; Revised 14 February 2011; Accepted 25 February 2011

Academic Editor: Ilmar Ferreira Santos

Copyright (c) 2011 Chun-xia Dou et al. This is an open access article distributed under the Creative Commons Attribution License, which permits unrestricted use, distribution, and reproduction in any medium, provided the original work is properly cited.

\begin{abstract}
A delay-dependent robust fuzzy control approach is developed for a class of nonlinear uncertain interconnected time delay large systems in this paper. First, an equivalent T-S fuzzy model is extended in order to accurately represent nonlinear dynamics of the large system. Then, a decentralized state feedback robust controller is proposed to guarantee system stabilization with a prescribed $H \infty$ disturbance attenuation level. Furthermore, taking into account the time delays in large system, based on a less conservative delay-dependent Lyapunov function approach combining with linear matrix inequalities (LMI) technique, some sufficient conditions for the existence of $H \infty$ robust controller are presented in terms of LMI dependent on the upper bound of time delays. The upper bound of time-delay and minimized $H \infty$ performance index can be obtained by using convex optimization such that the system can be stabilized and for all time delays whose sizes are not larger than the bound. Finally, the effectiveness of the proposed controller is demonstrated through simulation example.
\end{abstract}

\section{Introduction}

In recent years, interconnected large systems are very common in various fields, such as power systems, industrial processes, and spacecraft dynamics. In interconnected large systems, even if their subsystems have tractable models and interact with respective neighbors in a simple and predictable fashion, the entire systems often display rich and complex nonlinear behavior. Furthermore, due to the physical configuration and the high dimensionality, information transmission delays commonly exist in the interconnected large 
systems too. Over the past decade and before, the stability analysis and synthesis of large systems have been widely studied, and many different approaches have been proposed to stabilize these systems. For instance, in [1], general modeling and control method of large-scale systems is presented. Stabilization method of eigenvalue assignment for interconnected systems is proposed based on local feedback in [2]. References [3] and [4] display stability analysis and synthesis of large systems with time delay via Takagi-Sugeno fuzzy models, respectively. In [5] and [6], delay-dependent robust stability and LMI-based stability for fuzzy large-scale systems with time delays are researched, respectively. In [7], a nonlinear decentralized state feedback controller is presented for uncertain fuzzy time delay interconnected systems. The studies mentioned above indicate that in order to facilitate real engineering application, it is a prime requirement to transform the nonlinear models of large systems to linear models so as to enable them to be applicable into controller design. However, linearization technique and linear robust control method are obviously incompetent for controlling the complex nonlinear behavior of large systems, because the nonlinear behavior plays vital role during the transient state of the systems and often degrades system stability and dynamic performances. As an effective means, the nonlinear control schemes are also presented in the studies mentioned above. But, the nonlinear control schemes are so complicated that they are unfeasible for real applications. And they also post additional difficulties to controller design. So far, stabilization problem for complex large systems is still a baffling issue.

In general, due to the physical configuration and the high dimensionality, it is difficult to design a global centralized controller for large systems. And a centralized control is neither feasible nor economical. Due to decentralized control means that each subsystem is controlled independently on its locally available information, this control scheme is preferred in wide area control of large system. Many researchers have paid a great deal of attention on decentralized controllers for large systems. For instance, decentralized stabilizers for large interconnected power systems are designed in [8] and [9], respectively. In [10], robust stabilization is researched for interconnected large systems based on decentralized fuzzy control scheme. In [7] and [11], decentralized state feedback and decentralized guaranteed cost control are presented for large-scale systems, respectively. All the results mentioned above indicate that decentralized control scheme is more feasible and effective strategy for stabilization problem research of interconnected large systems.

Furthermore, in many cases, it is very complicated and difficult, even not possible to obtain the accurate nonlinear model of large systems. However, the nonlinearities should be precisely approximated as they play vital role during the transient state of the system. In order to facilitate real engineering application, in recent year, fuzzy logic model with if-then rules has become one of the most useful modeling approaches for complex systems $[12,13]$. The fuzzy-logic-based approach has the capability of approximating nonlinear processes to arbitrary accurate degrees so as to enable the fuzzy logic model to be applicable to system control $[14,15]$. Therefore, in order to effectively handle the stabilization problem for complex large systems, it is a straightforward idea to extend the T-S models that are interconnected, time delay, and uncertain to represent large systems. The modeling procedure of fuzzy logic model with if-then rules is given as follows: first, a nonlinear large system is decomposed into a number of interconnected subsystems. Each subsystem again is decomposed into a set of fuzzy regions, and in each region, local dynamic behavior of the subsystem is described by a T-S fuzzy model. Then, each subsystem dynamic is captured by a set of fuzzy implications that characterize local relations in the state space. Finally, the entire model of the nonlinear large system can be achieved by smoothly connecting the local linear model in each fuzzy 
subspace together via the membership functions. The method of T-S model can provide an effective solution to the control of complex large systems that are nonlinear, interconnected, time delay, and uncertain.

Studying the stabilization problem for large systems, it cannot avoid taking into consideration the transmission delays. The stabilization problem for time delay systems can be classified into two types: delay-independent stabilization and delay-dependent stabilization. The delay-independent stabilization for linear time delay system has been extensively studied by many researchers for the last decades. However, it is considered more conservative in general than the delay-dependent case, especially for time delay systems whose delay size is not actually small [4]. Many researchers also realize it necessary to develop delay-dependent stabilization method into control area of complex large systems that are nonlinear, interconnected, time delay, and uncertain. But, so far there are seldom relational contributions, mainly due to the complex nonlinear behavior in the systems bring huge complication to controller design.

In this paper, in order to effectively solve stabilization problem for complex large systems, we rigorously develop a less conservative delay-dependent robust stabilization method into the wide area control area of the large systems that are nonlinear, interconnected, time delay, and uncertain. The research is decomposed in several stages, starting with extending the T-S model that is interconnected, time delay, and uncertain to accurately represent nonlinear processes of the large systems. On the next stage, we propose a decentralized state-feedback fuzzy controller. Based on delay-dependent Lyapunov functional approach integrating the $H \infty$ robust control technique, some sufficient conditions for existence of the controller can be cast into the feasible problem of LMIs dependent on the upper bound size of time delays, by which the system can be robustly stabilized for all considered uncertainties and time delays whose sizes are not larger than their bounds. On the final stage, a procedure is also given to select suitable controller parameters that are optimal in the sense of minimizing the guaranteed $H \infty$ performance index by the modified generalized eigenvalue minimization problem technique in Matlab. The proposed method effectively handles the problems related to nonlinearities, time delays and uncertainties in large systems so as to enable controller to be more feasible and effective for real engineering application, which is a considerable contribution. The effectiveness of the proposed control method is demonstrated through simulation examples.

The paper is organized as follows. The problem is first formulated in Section 2. The delay-dependent stabilization problem via decentralized fuzzy controller is studied in Section 3. In Section 4, simulation case is provided to demonstrate the effectiveness of the proposed method. Finally, concluding remarks are given in Section 5.

\section{Problem Formulation}

Consider a class of nonlinear uncertain time delay large systems which are interconnected by $\mathbf{N}$ subsystems as follows:

$$
\begin{aligned}
\dot{\mathbf{x}}_{i}(t)= & f_{i}\left(\mathbf{x}_{i}(t), \mathbf{x}_{i}\left(t-\tau_{i i}\right), \mathbf{u}_{i}(t), \boldsymbol{\omega}_{i}(t)\right)+\Delta f_{i}\left(\mathbf{x}_{i}(t), \mathbf{x}_{i}\left(t-\tau_{i i}\right), \mathbf{u}_{i}(t), \boldsymbol{\omega}_{i}(t)\right) \\
& +\sum_{j=1, j \neq i}^{N}\left[f_{i j}\left(\mathbf{x}_{j}(t), \mathbf{x}_{j}\left(t-\tau_{i j}\right)\right)+\Delta f_{i j}\left(\mathbf{x}_{j}(t), \mathbf{x}_{j}\left(t-\tau_{i j}\right)\right)\right]
\end{aligned}
$$




$$
\begin{aligned}
& \mathbf{y}_{i}(t)=g_{i}\left(\mathbf{x}_{i}(t), \boldsymbol{\omega}_{i}(t)\right)+\Delta g_{i}\left(\mathbf{x}_{i}(t), \boldsymbol{\omega}_{i}(t)\right), \\
& \mathbf{x}_{i}(t)=\varphi_{i}(t), \quad t \in\left[-\tau_{i i}, 0\right], \quad \mathbf{x}_{j}(t)=\varphi_{j}(t), \quad t \in\left[-\tau_{i j}, 0\right], 0<\tau_{i i}, \tau_{i j} \leq \bar{\tau},
\end{aligned}
$$

where $\mathbf{x}_{i}(t) \in R^{n_{i}}, \mathbf{x}_{j}(t) \in R^{n_{j}}$ are state vectors of the $i$ th and $j$ th subsystems, respectively, $i, j=1,2, \ldots, N . \mathbf{u}_{i}(t) \in R^{m_{i}}$ is control input. $\mathbf{y}_{i}(t)$ and $\boldsymbol{\omega}_{i}(t)$ are controlled output and external disturbance of the $i$ th subsystem, respectively. $f_{i}(\cdot), g_{i}(\cdot), \Delta f_{i}(\cdot), \Delta g_{i}(\cdot), f_{i j}(\cdot)$, and $\Delta f_{i j}(\cdot)$ are the smooth nonlinear functions. $f_{i j}(\cdot)$ and $\Delta f_{i j}(\cdot)$ denote the interconnection functions between the $i$ th and $j$ th subsystem, $\Delta f_{i}(\cdot), \Delta g_{i}(\cdot)$, and $\Delta f_{i j}(\cdot)$ represent uncertainties in the $i$ th subsystem model. $\tau_{i i}$ and $\tau_{i j}$ are the constant but unknown delay in the states, $\bar{\tau}$ is the upper bound value of all time delays.

Here, the T-S fuzzy model is extended to represent the system (2.1). The model is described by fuzzy if-then rules in the following form.

The $k$ th rule of T-S fuzzy model for the $i$ th subsystem: $R_{i}^{k}$ : If $z_{i 1}(t)$ is $F_{k 1}, \ldots$, and $z_{i g_{i}}(t)$ is $F_{k g_{i}}$, Then,

$$
\begin{aligned}
\dot{\mathbf{x}}_{i}(t)= & \mathbf{A}_{i k} \mathbf{x}_{i}(t)+\mathbf{A}_{d i k} \mathbf{x}_{i}\left(t-\tau_{i i}\right)+\mathbf{B}_{1 i k} \mathbf{u}_{i}(t)+\mathbf{B}_{2 i k} \boldsymbol{\omega}_{i}(t)+\Delta \mathbf{A}_{i k} \mathbf{x}_{i}(t) \\
& +\Delta \mathbf{A}_{d i k} \mathbf{x}_{i}\left(t-\tau_{i i}\right)+\Delta \mathbf{B}_{1 i k} \mathbf{u}_{i}(t)+\Delta \mathbf{B}_{2 i k} \boldsymbol{\omega}_{i}(t) \\
& +\sum_{j=1, j \neq i}^{N}\left[\mathbf{A}_{i j k} \mathbf{x}_{j}(t)+\mathbf{A}_{d i j k} \mathbf{x}_{j}\left(t-\tau_{i j}\right)+\Delta \mathbf{A}_{i j k} \mathbf{x}_{j}(t)+\Delta \mathbf{A}_{d i j k} \mathbf{x}_{j}\left(t-\tau_{i j}\right)\right], \\
\mathbf{y}_{i}(t)= & \mathbf{C}_{i k} \mathbf{x}_{i}(t)+\Delta \mathbf{C}_{i k} \mathbf{x}_{i}(t)+\mathbf{D}_{i k} \boldsymbol{\omega}_{i}(t)+\Delta \mathbf{D}_{i k} \boldsymbol{\omega}_{i}(t),
\end{aligned}
$$

where $k=1,2, \ldots, l, l$ is the number of if-then rules $F_{k 1}, \ldots, F_{k g_{i}}$ are the fuzzy sets; $z_{i 1}(t), \ldots, z_{i g_{i}}(t)$ are the premise variables. $\mathbf{A}_{i k}, \mathbf{A}_{d i k}, \mathbf{A}_{i j k}, \mathbf{A}_{d i j k}, \mathbf{B}_{1 i k}, \mathbf{B}_{2 i k}, \mathbf{C}_{i k}$, and $\mathbf{D}_{i k}$ are constant coefficient matrices with appropriate dimensions. $\Delta \mathbf{A}_{i k}, \Delta \mathbf{A}_{d i k}, \Delta \mathbf{A}_{i j k}, \Delta \mathbf{A}_{d i j k}, \Delta \mathbf{B}_{1 i k}$, $\Delta \mathbf{B}_{2 i k}, \Delta \mathbf{C}_{i k}$, and $\Delta \mathbf{D}_{i k}$ are uncertain matrices with appropriate dimensions. form:

Then, the overall fuzzy model of the $i$ th subsystem can be rearranged in the following

$$
\begin{aligned}
& \dot{\mathbf{x}}_{i}(t)=\sum_{k=1}^{l} h_{k}\left(z_{i}(t)\right)\left\{\mathbf{A}_{i k} \mathbf{x}_{i}(t)+\mathbf{A}_{d i k} \mathbf{x}_{i}\left(t-\tau_{i i}\right)+\mathbf{B}_{1 i k} \mathbf{u}_{i}(t)+\mathbf{B}_{2 i k} \boldsymbol{\omega}_{i}(t)+\Delta \mathbf{A}_{i k} \mathbf{x}_{i}(t)\right. \\
& +\Delta \mathbf{A}_{d i k} \mathbf{x}_{i}\left(t-\tau_{i i}\right)+\Delta \mathbf{B}_{1 i k} \mathbf{u}_{i}(t)+\Delta \mathbf{B}_{2 i k} \boldsymbol{\omega}_{i}(t) \\
& \left.+\sum_{j=1, j \neq i}^{N}\left[\mathbf{A}_{i j k} \mathbf{x}_{j}(t)+\mathbf{A}_{d i j k} \mathbf{x}_{j}\left(t-\tau_{i j}\right)+\Delta \mathbf{A}_{i j k} \mathbf{x}_{j}(t)+\Delta \mathbf{A}_{d i j k} \mathbf{x}_{j}\left(t-\tau_{i j}\right)\right]\right\}, \\
& \mathbf{y}_{i}(t)=\sum_{k=1}^{l} h_{k}\left(z_{i}(t)\right)\left\{\mathbf{C}_{i k} \mathbf{x}_{i}(t)+\Delta \mathbf{C}_{i k} \mathbf{x}_{i}(t)+\mathbf{D}_{i k} \boldsymbol{\omega}_{i}(t)+\Delta \mathbf{D}_{i k} \boldsymbol{\omega}_{i}(t)\right\}, \\
& \mathbf{x}_{i}(t)=\varphi_{i}(t), \quad t \in\left[-\tau_{i i}, 0\right], \quad \mathbf{x}_{j}(t)=\varphi_{j}(t), \quad t \in\left[-\tau_{i j}, 0\right],
\end{aligned}
$$


where $\mu_{k}\left(z_{i}(t)\right)=\prod_{j=1}^{g_{i}} F_{k j}\left(z_{i j}(t)\right), h_{k}\left(z_{i}(t)\right)=\mu_{k}\left(z_{i}(t)\right) / \sum_{k=1}^{l} \mu_{k}\left(z_{i}(t)\right), z_{i}(t)=\left[z_{i 1}(t)\right.$, $\left.z_{i 2}(t), \ldots, z_{i g_{i}}(t)\right]$, and $F_{k g_{i}}\left(z_{i g_{i}}(t)\right)$ is the grade of membership of $z_{i g_{i}}(t)$ in $F_{k g_{i}}$. Assume that $\mu_{k}(z(t)) \geq 0$, for $k=1,2, \ldots, l$ and all $t$. Then, $h_{k}(z(t)) \geq 0$, for $k=1,2, \ldots, l$, and $\sum_{k=1}^{l} h_{k}(z(t))=1$.

\section{Delay-Dependent Robust Stabilization via Decentralized Fuzzy Control}

In the following, if not stated, matrices are assumed to have compatible dimensions. The identify and zero matrices are denoted by I and $\mathbf{0}$, respectively. The notation $*$ always denotes the symmetric block in one symmetric matrix. The standard notation $>(<)$ is used to denote the positive (negative) definite ordering of matrices. Inequality $\mathbf{X}>\mathrm{Y}$ shows that the matrix $\mathbf{X}-\mathbf{Y}$ is positive definite.

First, the decentralized fuzzy controller is designed to deal with the robust stabilization problem for the above system in the following form:

$$
R_{i}^{k} \text { : If } z_{i 1}(t) \text { is } F_{k 1}, \ldots \text {, and } z_{i g_{i}}(t) \text { is } F_{k g_{i}} \text {, then } \mathbf{u}_{i}(t)=\mathbf{K}_{i k} \mathbf{x}_{i}(t) .
$$

Then, the overall controller has the form

$$
\mathbf{u}_{i}(t)=\sum_{k=1}^{l} h_{k}\left(z_{i}(t)\right) \mathbf{K}_{i k} \mathbf{x}_{i}(t)
$$

Substituting (3.2) into (2.3) yields the ith closed-loop decentralized controlled subsystem as follows:

$$
\begin{aligned}
& \dot{\mathbf{x}}_{i}(t)=\sum_{k=1}^{l} \sum_{m=1}^{l} h_{k}\left(z_{i}(t)\right) h_{m}\left(z_{i}(t)\right) \\
& \times\left\{\left(\mathbf{A}_{i k}+\mathbf{B}_{i k} \mathbf{K}_{i m}\right) \mathbf{x}_{i}(t)+\mathbf{A}_{d i k} \mathbf{x}_{i}\left(t-\tau_{i i}\right)+\Delta \mathbf{A}_{d i k} \mathbf{x}_{i}\left(t-\tau_{i i}\right)+\mathbf{B}_{2 i k} \boldsymbol{\omega}_{i}(t)\right. \\
& +\Delta \mathbf{B}_{2 i k} \boldsymbol{\omega}_{i}(t)+\left(\Delta \mathbf{A}_{i k}+\Delta \mathbf{B}_{i k} \mathbf{K}_{i m}\right) \mathbf{x}_{i}(t) \\
& \left.+\sum_{j=1, j \neq i}^{N}\left[\mathbf{A}_{i j k} \mathbf{x}_{j}(t)+\mathbf{A}_{d i j k} \mathbf{x}_{j}\left(t-\tau_{i j}\right)+\Delta \mathbf{A}_{i j k} \mathbf{x}_{j}(t)+\Delta \mathbf{A}_{d i j k} \mathbf{x}_{j}\left(t-\tau_{i j}\right)\right]\right\}, \\
& \mathbf{y}_{i}(t)=\sum_{k=1}^{l} h_{k}\left(z_{i}(t)\right)\left[\mathbf{C}_{i k} \mathbf{x}_{i}(t)+\Delta \mathbf{C}_{i k} \mathbf{x}_{i}(t)+\mathbf{D}_{i k} \boldsymbol{\omega}_{i}(t)+\Delta \mathbf{D}_{i k} \boldsymbol{\omega}_{i}(t)\right], \\
& \mathbf{x}_{i}(t)=\varphi_{i}(t), \quad t \in\left[-\tau_{i i}, 0\right], \quad \mathbf{x}_{j}(t)=\varphi_{j}(t), \quad t \in\left[-\tau_{i j}, 0\right] .
\end{aligned}
$$

Here, the uncertainties in the system are represented by the uncertain matrices with appropriate dimensions. We may make the following assumption concerning the uncertain matrices. 
Assumption 1 (see [16]). The uncertainties considered here are norm-bounded in the form

$$
\begin{aligned}
{\left[\begin{array}{llllllll}
\Delta \mathbf{A}_{i k} & \Delta \mathbf{B}_{1 i k} & \Delta \mathbf{A}_{d i k} & \Delta \mathbf{A}_{i j k} & \Delta \mathbf{A}_{d i j k} & \Delta \mathbf{B}_{2 i k} & \Delta \mathbf{C}_{i k} & \Delta \mathbf{D}_{i k}
\end{array}\right] } \\
=\mathbf{H}_{i k} \mathbf{F}_{i k}(t)\left[\begin{array}{llllllll}
\mathbf{E}_{1 i k} & \mathbf{E}_{2 i k} & \mathbf{E}_{3 i k} & \mathbf{E}_{4 i j k} & \mathbf{E}_{5 i j k} & \mathbf{E}_{6 i k} & \mathbf{E}_{7 i k} & \mathbf{E}_{8 i k}
\end{array}\right],
\end{aligned}
$$

where $\mathbf{H}_{i k}, \mathbf{E}_{1 i k}, \mathbf{E}_{2 i k}, \mathbf{E}_{3 i k}, \mathbf{E}_{4 i j k}, \mathbf{E}_{5 i j k}, \mathbf{E}_{6 i k}, \mathbf{E}_{7 i k}$, and $\mathbf{E}_{8 i k}$ are the real constant matrices known of appropriate dimensions and $\mathbf{F}_{i k}(t)$ is an unknown matrix function with Lebesguemeasurable elements and satisfies $\mathbf{F}_{i k}^{T}(t) \mathbf{F}_{i k}(t) \leq \mathbf{I}$.

Accordingly, substituting (3.4) into (3.3) yields the $i$ th closed-loop decentralized controlled subsystem as follows:

$$
\begin{aligned}
& \dot{\mathbf{x}}_{i}(t)= \sum_{k=1}^{l} \sum_{m=1}^{l} h_{k}\left(z_{i}(t)\right) h_{m}\left(z_{i}(t)\right) \\
& \times\left\{\left[\mathbf{A}_{i k}+\mathbf{B}_{1 i k} \mathbf{K}_{i m}+\mathbf{H}_{i k} \mathbf{F}_{i k}(t)\left(\mathbf{E}_{1 i k}+\mathbf{E}_{2 i k} \mathbf{K}_{i m}\right)\right] \mathbf{x}_{i}(t)+\left(\mathbf{A}_{d i k}+\mathbf{H}_{i k} \mathbf{F}_{i k}(t) \mathbf{E}_{3 i k}\right) \mathbf{x}_{i}\left(t-\tau_{i i}\right)\right. \\
&+\sum_{j=1, j \neq i}^{N}\left[\left(\mathbf{A}_{i j k}+\mathbf{H}_{i k} \mathbf{F}_{i k}(t) \mathbf{E}_{4 i j k}\right) \mathbf{x}_{j}(t)+\left(\mathbf{A}_{d i j k}+\mathbf{H}_{i k} \mathbf{F}_{i k}(t) \mathbf{E}_{5 i j k}\right) \mathbf{x}_{j}\left(t-\tau_{i j}\right)\right] \\
&\left.+\left(\mathbf{B}_{2 i k}+\mathbf{H}_{i k} \mathbf{F}_{i k}(t) \mathbf{E}_{6 i k}\right) \boldsymbol{\omega}_{i}(t)\right\}, \\
& \mathbf{y}_{i}(t)=\sum_{k=1}^{l} h_{k}\left(z_{i}(t)\right)\left[\left(\mathbf{C}_{i k}+\mathbf{H}_{i k} \mathbf{F}_{i k}(t) \mathbf{E}_{7 i k}\right) \mathbf{x}_{i}(t)+\left(\mathbf{D}_{i k}+\mathbf{H}_{i k} \mathbf{F}_{i k}(t) \mathbf{E}_{8 i k}\right) \boldsymbol{\omega}_{i}(t)\right] .
\end{aligned}
$$

Equation (3.5) can be rearranged in the following form:

$$
\begin{aligned}
\dot{\mathbf{x}}_{i}(t)= & \sum_{k=1}^{l} \sum_{m=1}^{l} h_{k}\left(z_{i}(t)\right) h_{m}\left(z_{i}(t)\right) \\
& \times\left\{\left[\mathbf{A}_{i k}+\mathbf{B}_{1 i k} \mathbf{K}_{i m}+\mathbf{H}_{i k} \mathbf{F}_{i k}(t)\left(\mathbf{E}_{1 i k}+\mathbf{E}_{2 i k} \mathbf{K}_{i m}\right)+\mathbf{A}_{d i k}+\mathbf{H}_{i k} \mathbf{F}_{i k}(t) \mathbf{E}_{3 i k}\right] \mathbf{x}_{i}(t)\right. \\
& -\left(\mathbf{A}_{d i k}+\mathbf{H}_{i k} \mathbf{F}_{i k}(t) \mathbf{E}_{3 i k}\right) \int_{t-\tau_{i i}}^{t} \dot{\mathbf{x}}_{i}(\alpha) d \alpha \\
& +\sum_{j=1, j \neq i}^{N}\left[\left(\mathbf{A}_{i j k}+\mathbf{H}_{i k} \mathbf{F}_{i k}(t) \mathbf{E}_{4 i j k}\right) \mathbf{x}_{j}(t)+\left(\mathbf{A}_{d i j k}+\mathbf{H}_{i k} \mathbf{F}_{i k}(t) \mathbf{E}_{5 i j k}\right) \mathbf{x}_{j}\left(t-\tau_{i j}\right)\right]
\end{aligned}
$$




$$
\begin{gathered}
\left.+\left(\mathbf{B}_{2 i k}+\mathbf{H}_{i k} \mathbf{F}_{i k}(t) \mathbf{E}_{6 i k}\right) \boldsymbol{\omega}_{i}(t)\right\}, \\
\mathbf{y}_{i}(t)=\sum_{k=1}^{l} h_{k}\left(z_{i}(t)\right)\left[\left(\mathbf{C}_{i k}+\mathbf{H}_{i k} \mathbf{F}_{i k}(t) \mathbf{E}_{7 i k}\right) \mathbf{x}_{i}(t)+\left(\mathbf{D}_{i k}+\mathbf{H}_{i k} \mathbf{F}_{i k}(t) \mathbf{E}_{8 i k}\right) \boldsymbol{\omega}_{i}(t)\right] .
\end{gathered}
$$

Let us define a delay-independent Lyapunov function for the system (3.6) as

$$
\begin{gathered}
V_{i}(t)=V_{i 1}(t)+V_{i 2}(t)+V_{i 3}(t), \\
V_{i 1}(t)=\mathbf{x}_{i}^{T}(t) \mathbf{P}_{i i} \mathbf{x}_{i}(t), \\
V_{i 2}(t)=\int_{t-\tau_{i i}}^{t} \mathbf{x}_{i}^{T}(\tau) \mathbf{S}_{i i} \mathbf{x}_{i}(\tau) d \tau, \\
V_{i 3}(t)=\int_{-\tau_{i i}}^{0} \int_{t+\beta}^{t} \dot{\mathbf{x}}_{i}^{T}(\alpha) \mathbf{Z}_{i i} \dot{\mathbf{x}}_{i}(\alpha) d \alpha d \beta,
\end{gathered}
$$

where $\mathbf{P}_{i i}, \mathbf{S}_{i i}, \mathbf{Z}_{i i}$ are symmetric positive definite weighting matrices.

Furthermore, for robust stabilization purpose, $H \infty$ performance related to the controlled output is proposed in the following form:

$$
J_{i}=\int_{0}^{t_{f}}\left[\rho_{i}^{-1} \mathbf{y}_{i}^{T}(t) \mathbf{y}_{i}(t)-\rho_{i} \boldsymbol{\omega}_{i}^{T}(t) \boldsymbol{\omega}_{i}(t)\right] d t
$$

Considering the initial condition, the $H \infty$ performance can be rewritten as follows:

$$
\int_{0}^{t_{f}} \mathbf{y}_{i}^{T}(t) \mathbf{y}_{i}(t) d t \leq \rho_{i}^{2} \int_{0}^{t_{f}} \boldsymbol{\omega}_{i}^{T}(t) \boldsymbol{\omega}_{i}(t) d t+V_{i}(0)
$$

where $\rho_{i}$ is a prescribed attenuation level.

Remark 3.1. The physical meaning of (3.9) is that the every time delay interconnected subsystem is stable in the sense of Lyapunov, and the effect of any $\boldsymbol{\omega}_{i}(t)$ on output $\mathbf{y}_{i}(t)$ must be attenuated below a desire level $\rho_{i}$ from the viewpoint of energy. No matter what $\boldsymbol{\omega}_{i}(t)$ is, the $L_{2}$ gain from $\boldsymbol{\omega}_{i}(t)$ to $\mathbf{y}_{i}(t)$ must be equal to or less than a prescribed value $\rho_{i}^{2}$. The $H \infty$ performance with a prescribed attenuation level is useful for a robust design without knowledge of $\boldsymbol{\omega}_{i}(t)$.

Remark 3.2. The purpose of this study is to guarantee stability of the system (3.6) via the decentralized fuzzy controller (3.2). Thereafter, the attenuation level $\rho_{i}$ can also be minimized so that the $H \infty$ performance as (3.9) is reduced as small as possible for all $\boldsymbol{\omega}_{i}(t)$ and $i=$ $1,2, \ldots, N$. 
The following lemmas will play important roles in obtaining results in this paper. We show them as follows.

Lemma 3.3 (see [16]). For all vectors $a$ and $b$ matrices $\mathbf{N}, \mathbf{X}, \mathbf{Y}$, and $\mathbf{Z}$ with appropriate dimensions, if $\left[\begin{array}{cc}\mathbf{X} & \mathbf{Y} \\ \mathbf{Y}^{T} & \mathbf{Z}\end{array}\right] \geq 0$, one has

$$
-2 \mathbf{a}^{T} \mathbf{N b} \leq \inf _{X, Y, Z}\left\{\left[\begin{array}{l}
\mathbf{a} \\
\mathbf{b}
\end{array}\right]^{T}\left[\begin{array}{cc}
\mathbf{X} & \mathbf{Y}-\mathbf{N} \\
\mathbf{Y}^{T}-\mathbf{N}^{T} & \mathbf{Z}
\end{array}\right]\left[\begin{array}{l}
\mathbf{a} \\
\mathbf{b}
\end{array}\right]\right\},
$$

where $\mathbf{X}$ and $\mathbf{Z}$ are symmetrical positive matrices.

Lemma 3.4 (see [17]). For matrices (or vectors) Y, D, and $\mathbf{E}$ with appropriate dimensions, one has

$$
\mathbf{Y}+\mathbf{D F E}+\mathbf{E}^{T} \mathbf{F}^{T} \mathbf{D}^{T}<0
$$

where $\mathbf{Y}$ is a symmetric matrix. For all $\mathbf{F}$ satisfy $\mathbf{F}^{\mathbf{T}} \mathbf{F} \leq \mathbf{I}$, if and only if a set of scalar quantity $\varepsilon>0$ exist, one gains

$$
\mathbf{Y}+\varepsilon \mathbf{D D}^{T}+\varepsilon^{-1} \mathbf{E}^{\mathrm{T}} \mathbf{E}<0
$$

Based on delay-dependent Lyapunov function integrating the $H \infty$ performance, some sufficient conditions for the system robust stabilization are shown in Theorem 3.5.

Theorem 3.5. The interconnected uncertain time delay fuzzy system (3.6) is asymptotically stable via the decentralized fuzzy controller in (3.2) and satisfies the Ho control performance in (3.9) for a prescribed $\rho_{i}^{2}$ when $\boldsymbol{\omega}_{i}(t) \neq 0$, only if $\mathbf{P}_{i i}=\mathbf{P}_{i i}^{T}>0, \mathbf{S}_{i i}=\mathbf{S}_{i i}^{T}>0, \mathbf{Z}_{i i}=\mathbf{Z}_{i i}^{T}>0$, are the common solutions of the following matrix inequalities for $i, j=1,2, \ldots, N(j \neq i)$ and $k, m=1,2, \ldots, l\left[\begin{array}{cc}\mathbf{X}_{i i} & \mathbf{Y}_{i i} \\ \mathbf{Y}_{i i}{ }^{T} & \mathbf{Z}_{i i}\end{array}\right] \geq 0$, and

$$
\left[\begin{array}{ccccccc}
\mathbf{Z}_{i j k m} & \widehat{\mathbf{Z}}_{i k} & \widehat{\mathbf{Z}}_{i j k} & \overline{\mathbf{Z}}_{i j k} & \breve{\mathbf{Z}}_{i k} & \mathbf{\Phi}_{i k m}^{T} & \left(\mathbf{C}_{i k}+\mathbf{H}_{i k} \mathbf{F}_{i k}(t) \mathbf{E}_{7 i k}\right)^{T} \\
* & -\mathbf{S}_{i i} & 0 & 0 & 0 & {\left[\mathbf{A}_{d i k}+\mathbf{H}_{i k} \mathbf{F}_{i k}(t) \mathbf{E}_{3 i k}\right]^{T}} & 0 \\
* & * & 0 & 0 & 0 & {\left[\mathbf{A}_{i j k}+\mathbf{H}_{i k} \mathbf{F}_{i k}(t) \mathbf{E}_{4 i j k}\right]^{T}} & 0 \\
* & * & * & 0 & 0 & {\left[\mathbf{A}_{d i j k}+\mathbf{H}_{i k} \mathbf{F}_{i k}(t) \mathbf{E}_{5 i j k}\right]^{T}} & 0 \\
* & * & * & * & -\rho_{i}^{2} & {\left[\mathbf{B}_{2 i k}+\mathbf{H}_{i k} \mathbf{F}_{i k}(t) \mathbf{E}_{6 i k}\right]^{T}} & \left(\mathbf{D}_{i k}+\mathbf{H}_{i k} \mathbf{F}_{i k}(t) \mathbf{E}_{8 i k}\right)^{T} \\
* & * & * & * & * & -\bar{\tau}^{-1} \mathbf{Z}_{i i}^{-1} & 0 \\
* & * & * & * & * & * & -\mathbf{I}
\end{array}\right] \leq 0,
$$


where

$$
\begin{aligned}
& \mathbf{Z}_{i k m}= \mathbf{P}_{i i}\left[\mathbf{A}_{i k}+\mathbf{B}_{1 i k} \mathbf{K}_{i m}+\mathbf{H}_{i k} \mathbf{F}_{i k}(t)\left(\mathbf{E}_{1 i k}+\mathbf{E}_{2 i k} \mathbf{K}_{i m}\right)\right] \\
&+\left[\mathbf{A}_{i k}+\mathbf{B}_{1 i k} \mathbf{K}_{i m}+\mathbf{H}_{i k} \mathbf{F}_{i k}(t)\left(\mathbf{E}_{1 i k}+\mathbf{E}_{2 i k} \mathbf{K}_{i m}\right)\right]^{T} \mathbf{P}_{i i}+\bar{\tau} \mathbf{X}_{i i}+\mathbf{Y}_{i i}+\mathbf{Y}_{i i}^{T}+\mathbf{S}_{i i}, \\
& \widehat{\mathbf{Z}}_{i k}= \mathbf{P}_{i i}\left(\mathbf{A}_{d i k}+\mathbf{H}_{i k} \mathbf{F}_{i k}(t) \mathbf{E}_{3 i k}\right)-\mathbf{Y}_{i i}, \quad \widehat{\mathbf{Z}}_{i j k}=\mathbf{P}_{i i}\left(\mathbf{A}_{i j k}+\mathbf{H}_{i k} \mathbf{F}_{i k}(t) \mathbf{E}_{4 i j k}\right) \\
& \overline{\mathbf{Z}}_{i j k}= \mathbf{P}_{i i}\left(\mathbf{A}_{d i j k}+\mathbf{H}_{i k} \mathbf{F}_{i k}(t) \mathbf{E}_{5 i j k}\right), \quad \breve{\mathbf{Z}}_{i k}=\mathbf{P}_{i i}\left(\mathbf{B}_{2 i k}+\mathbf{H}_{i k} \mathbf{F}_{i k}(t) \mathbf{E}_{6 i k}\right), \\
& \Phi_{i k m}=\mathbf{A}_{i k}+\mathbf{B}_{1 i k} \mathbf{K}_{i m}+\mathbf{H}_{i k} \mathbf{F}_{i k}(t)\left(\mathbf{E}_{1 i k}+\mathbf{E}_{2 i k} \mathbf{K}_{i m}\right) .
\end{aligned}
$$

The proof is introduced in Appendix A.

There is an unknown matrix function $\mathbf{F}_{i k}(t)$ in (3.13), let us deal with the parameter uncertainty problem as follow.

Theorem 3.6. The uncertain interconnected fuzzy system with time delays (3.6) is asymptotically stable via the decentralized fuzzy controller in (3.2) and satisfies the Ho performance in (3.9) with a prescribed $\rho_{i}^{2}$, only if a set of scalar quantity $\varepsilon_{i}>0, \gamma_{i}>0$ and matrices $\mathbf{P}_{i i}=\mathbf{P}_{i i}^{T}>0, \mathbf{S}_{i i}=\mathbf{S}_{i i}^{T}>0$, $\mathbf{Z}_{i i}=\mathbf{Z}_{i i}^{T}>0$ are the common solutions of the following matrix inequalities: $\left[\begin{array}{c}\mathbf{X}_{i i} \mathbf{Y}_{i i} \\ \mathbf{Y}_{i i} \mathbf{Z}_{i i}\end{array}\right] \geq 0$, and

$$
\left[\begin{array}{ccccc}
\mathbf{Z}_{i k m}^{\prime} & \widehat{\mathbf{Z}}_{i k}^{\prime} & \widehat{\mathbf{Z}}_{i j k}^{\prime} & \overline{\mathbf{Z}}_{i j k}^{\prime} & \breve{\mathbf{Z}}_{i k}^{\prime} \\
* & -\mathbf{S}_{i i} & 0 & 0 & 0 \\
* & * & 0 & 0 & 0 \\
* & * & * & 0 & 0 \\
* & * & * & * & -\rho_{i}^{2} \mathbf{I} \\
* & * & * & * & * \\
* & * & * & * & * \\
* & * & * & * & * \\
* & * & * & * & * \\
* & * & * & * & * \\
* & * & * & * & *
\end{array}\right.
$$

$$
\left.\begin{array}{cccccc}
\left(\mathbf{A}_{i k}+\mathbf{B}_{1 i k} \mathbf{K}_{i m}\right)^{T} & \mathbf{C}_{i k}^{T} & \mathbf{P}_{i i} \mathbf{H}_{i k} & 0 & \left(\mathbf{E}_{1 i k}+\mathbf{E}_{2 i k} \mathbf{K}_{i m}\right)^{T} & \mathbf{E}_{5 i k}^{T} \\
\mathbf{A}_{d i k}^{T} & 0 & 0 & 0 & \mathbf{E}_{3 i k}^{T} & 0 \\
\mathbf{A}_{i j k}^{T} & 0 & 0 & 0 & \mathbf{E}_{4 i j k}^{T} & 0 \\
\mathbf{A}_{d i j k}^{T} & 0 & 0 & 0 & \mathbf{E}_{5 i j k}^{T} & 0 \\
\mathbf{B}_{2 i k}^{T} & \mathbf{D}_{i k}^{T} & 0 & 0 & \mathbf{E}_{6 i k}^{T} & \mathbf{E}_{6 i k}^{T} \\
-\bar{\tau}^{-1} \mathbf{Z}_{i i}^{-1} & 0 & \mathbf{H}_{i k} & 0 & 0 & 0 \\
* & -\mathbf{I} & 0 & \mathbf{H}_{i k} & 0 & 0 \\
* & * & -\varepsilon_{i}^{-1} \mathbf{I} & 0 & 0 & 0 \\
* & * & * & -\gamma_{i}^{-1} \mathbf{I} & 0 & 0 \\
* & * & * & * & -\varepsilon_{i} \mathbf{I} & 0 \\
* & * & * & * & * & -\gamma_{i} \mathbf{I}
\end{array}\right] \leq 0,
$$

where

$$
\begin{gathered}
\mathbf{Z}_{i k m}^{\prime}=\mathbf{P}_{i i}\left[\mathbf{A}_{i k}+\mathbf{B}_{1 i k} \mathbf{K}_{i m}\right]+\left[\mathbf{A}_{i k}+\mathbf{B}_{1 i k} \mathbf{K}_{i m}\right]^{T} \mathbf{P}_{i i}+\bar{\tau} \mathbf{X}_{i i}+\mathbf{Y}_{i i}+\mathbf{Y}_{i i}^{T}+\mathbf{S}_{i i}, \\
\widehat{\mathbf{Z}}_{i k}^{\prime}=\mathbf{P}_{i i} \mathbf{A}_{d i k}-\mathbf{Y}_{i i}, \quad \widetilde{\mathbf{Z}}_{i j k}^{\prime}=\mathbf{P}_{i i} \mathbf{A}_{i j k}, \quad \overline{\mathbf{Z}}_{i j k}^{\prime}=\mathbf{P}_{i i} \mathbf{A}_{d i j k}, \quad \breve{\mathbf{Z}}_{i k}^{\prime}=\mathbf{P}_{i i} \mathbf{B}_{2 i k} .
\end{gathered}
$$

The proof of Theorem 3.6 is easy to obtain. By Lemma 3.4 and Schur decomposition [19], the inequality (3.13) is equivalent to the inequality (3.15). 
The inequality (3.15) just relates into the upper bound size of time delays and determined norm-bounds of uncertainties. However it is not linear, let us left multiply and right multiply matrix $\mathbf{W}_{i i}=\operatorname{diag}\left\{\mathbf{p}_{i i}^{-1}, \mathbf{I}, \mathbf{I}, \mathbf{I}, \mathbf{I}, \mathbf{I}, \mathbf{I}, \mathbf{I}, \mathbf{I}, \mathbf{I}, \mathbf{I}\right\}$, (3.15) is equivalent to LMI (3.17)

$$
\left[\begin{array}{ccccccccccc}
\mathbf{Z}_{i k m}^{\prime \prime} & \widehat{\mathbf{Z}}_{i k}^{\prime \prime} & \mathbf{A}_{i j k} & \mathbf{A}_{d i j k} & \mathbf{B}_{2 i k} & \left(\mathbf{A}_{i k} \overline{\mathbf{X}}_{i i}+\mathbf{B}_{1 i k} \overline{\mathbf{Y}}_{\mathrm{i} m}\right)^{T} & \left(\mathbf{C}_{i k} \overline{\mathbf{X}}_{i i}\right)^{T} & \mathbf{H}_{i k} & 0 & \left(\mathbf{E}_{1 i k} \overline{\mathbf{X}}_{i i}+\mathbf{E}_{2 i k} \overline{\mathbf{Y}}_{\mathrm{i} m}\right)^{T} & \left(\mathbf{E}_{5 i k} \overline{\mathbf{X}}_{i i}\right)^{T} \\
* & -\mathbf{S}_{i i} & 0 & 0 & 0 & \mathbf{A}_{d i k}^{T} & 0 & 0 & 0 & \mathbf{E}_{3 i k}^{T} & 0 \\
* & * & 0 & 0 & 0 & \mathbf{A}_{i j k}^{T} & 0 & 0 & 0 & \mathbf{E}_{4 i k k}^{T} & 0 \\
* & * & * & 0 & 0 & \mathbf{A}_{d i j k}^{T} & 0 & 0 & 0 & \mathbf{E}_{5 i j k}^{T} & 0 \\
* & * & * & * & -\rho_{i}^{2} \mathbf{I} & \mathbf{B}_{2 i k}^{T} & \mathbf{D}_{i k}^{T} & 0 & 0 & \mathbf{E}_{6 i k}^{T} & \mathbf{E}_{6 i k}^{T} \\
* & * & * & * & * & -\bar{\tau}^{-1} \mathbf{Z}_{i i}^{-1} & 0 & \mathbf{H}_{i k} & 0 & 0 & 0 \\
* & * & * & * & * & * & -\mathbf{I} & 0 & \mathbf{H}_{i k} & 0 & 0 \\
* & * & * & * & * & * & * & -\varepsilon_{i}^{-1} \mathbf{I} & 0 & 0 & 0 \\
* & * & * & * & * & * & * & * & -\gamma_{i}^{-1} \mathbf{I} & 0 & 0 \\
* & * & * & * & * & * & * & * & * & -\varepsilon_{i} \mathbf{I} & 0 \\
* & * & * & * & * & * & * & * & * & * & -\gamma_{i} \mathbf{I}
\end{array}\right]
$$

where

$$
\begin{gathered}
\mathbf{Z}_{i k m}^{\prime \prime}=\left[\mathbf{A}_{i k} \overline{\mathbf{X}}_{i i}+\mathbf{B}_{1 i k} \overline{\mathbf{Y}}_{\mathrm{i} m}\right]+\left[\mathbf{A}_{i k} \overline{\mathbf{X}}_{i i}+\mathbf{B}_{1 i k} \overline{\mathbf{Y}}_{\mathrm{i} m}\right]^{T}+\tau_{i i} \tilde{\mathbf{X}}_{i i}+\tilde{\mathbf{Y}}_{i i}+\tilde{\mathbf{Y}}_{i i}^{T}+\tilde{\mathbf{S}}_{i i}, \quad \tilde{\mathbf{X}}_{i i}=\mathbf{P}_{i i}^{-1} \mathbf{X}_{i i} \mathbf{P}_{i i}^{-1}, \\
\overline{\mathbf{X}}_{i i}=\mathbf{P}_{i i}^{-1}, \quad \tilde{\mathbf{Y}}_{i i}=\mathbf{P}_{i i}^{-1} \mathbf{Y}_{i i} \mathbf{P}_{i i}^{-1}, \quad \tilde{\mathbf{S}}_{i i}=\mathbf{P}_{i i}^{-1} \mathbf{S}_{i i} \mathbf{P}_{i i}^{-1}, \\
\overline{\mathbf{Y}}_{i m}=\mathbf{K}_{i m} \mathbf{P}_{i i}^{-1}, \quad \widehat{\mathbf{Z}}_{i k}^{\prime \prime}=\mathbf{A}_{d i k}-\overline{\mathbf{X}}_{i i} \mathbf{Y}_{i i}=\mathbf{A}_{d i k}-\widehat{\mathbf{Y}}_{i i} .
\end{gathered}
$$

Remark 3.7. To obtain better robust performance, the $H \infty$ control performance can be treated as the following minimization problem so that the $H \infty$ performance in (3.9) is reduced as small as possible

$J^{*}=\min \left[\alpha_{i}+\operatorname{Trace}\left(\mathbf{M}_{i}\right)+\operatorname{Trace}\left(\boldsymbol{\Theta}_{i}\right)+\operatorname{Trace}\left(\boldsymbol{\Lambda}_{i}\right)\right]$

s.t. (i) The inequality (3.17),

(ii) $\left[\begin{array}{ll}\mathbf{X}_{i i} & \mathbf{Y}_{i i} \\ \mathbf{Y}_{i i}^{T} & \mathbf{Z}_{i i}\end{array}\right] \geq 0, \quad$ (iii) $\left[\begin{array}{cc}-\alpha_{i} & \mathbf{x}_{i}^{T}(0) \\ \mathbf{x}_{i}(0) & -\mathbf{X}_{i i}\end{array}\right]<0$,

(iv) $\left[\begin{array}{cc}-\boldsymbol{\Theta}_{i} & \boldsymbol{\Pi}_{i}^{T} \\ \boldsymbol{\Pi}_{i} & -\mathbf{S}_{i i}^{-1}\end{array}\right]<0$,

(v) $\left[\begin{array}{cc}-\boldsymbol{\Lambda}_{i} & \boldsymbol{\Psi}_{i}^{T} \\ \boldsymbol{\Psi}_{i} & -\mathbf{Z}_{i i}^{-1}\end{array}\right]<0$,

(vi) $\left[\begin{array}{cc}-\mathbf{M}_{i} & \mathbf{N}_{i}^{T} \\ \mathbf{N}_{i} & -\rho_{i}^{-2} \mathbf{I}\end{array}\right]<0$, 


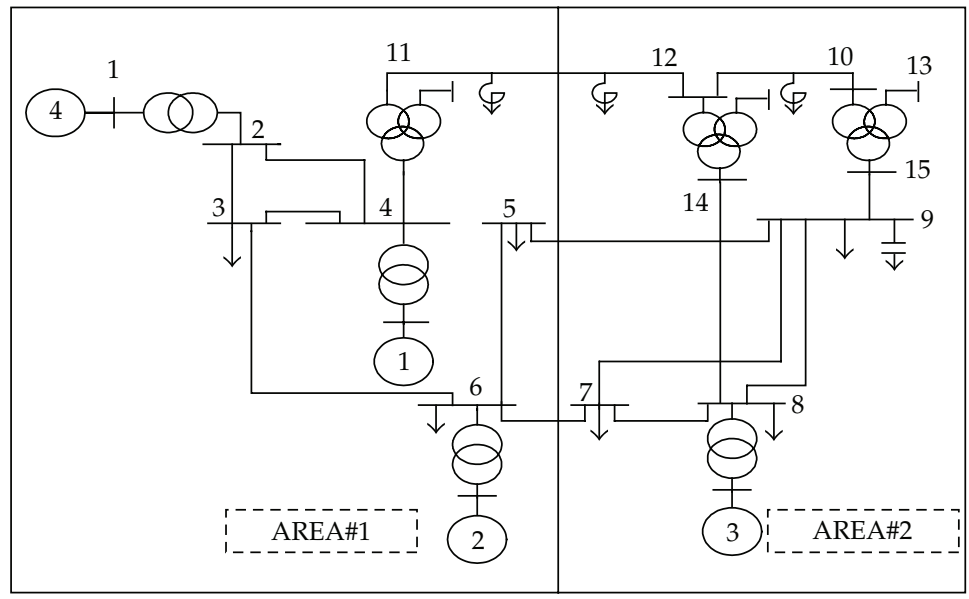

Figure 1: Four-machine interconnected power system.

where

$$
\begin{gathered}
\int_{0}^{t_{f}} \boldsymbol{\omega}_{i}^{T}(t) \boldsymbol{\omega}_{i}(t) d t=\mathbf{N}_{\mathbf{i}}^{\mathrm{T}} \mathbf{N}_{i}, \quad \int_{-\tau_{i i}}^{0} \varphi_{i}^{T}(t) \varphi_{i}(t) d t=\Pi_{\mathbf{i}}^{\mathrm{T}} \boldsymbol{\Pi}_{i}, \\
\int_{-\tau_{i i}}^{0} \int_{\beta}^{0} \dot{\varphi}_{i}^{T}(\alpha) \dot{\varphi}_{i}(\alpha) d \alpha d \beta=\Psi_{i}^{T} \Psi_{i} .
\end{gathered}
$$

The minimization problem in (3.19) can be transformed into LMI optimization problem. By convex optimization techniques of LMI via MATLAB tool boxes, the $H_{\infty}$ performance index is minimized, and the parameters of controller are obtained. Thereafter, the fuzzy decentralized controller in (3.2) is designed.

\section{Simulation Example}

We consider a four-machine power system that is a typical nonlinear interconnected uncertain large system as shown in Figure 1 to demonstrate the effectiveness of the proposed control method via the dynamic responses after faults. The dynamic equations of each synchronous generator are expressed as follows [19]:

$$
\begin{gathered}
\dot{\delta}_{i}(t)=\omega_{i}(t)-\omega_{0}, \\
\dot{\omega}_{i}(t)=\frac{\omega_{0}}{M_{i}} P_{m i}-\frac{D_{i}}{M_{i}}\left(\omega_{i}(t)-\omega_{0}\right)-\frac{\omega_{0}}{M_{i}} P_{e i}(t), \\
\dot{E}_{q i}^{\prime}(t)=-\frac{1}{T_{d o i}^{\prime}}\left[E_{q i}^{\prime}(t)+I_{d i}(t)\left(\mathbf{x}_{d i}-\mathbf{x}_{d i}^{\prime}\right)\right]+\frac{1}{T_{d o i}^{\prime}} V_{f i}(t), \\
\mathbf{y}_{i 1}(t)=\delta_{i}(t), \\
\mathbf{y}_{i 2}(t)=P_{e i}(t),
\end{gathered}
$$


Table 1: Synchronous generator parameters.

\begin{tabular}{lcccc}
\hline Parameter & Generator 1 & Generator 2 & Generator 3 & Generator 4 \\
\hline$x_{d}$ p.u. & 1.843 & 2.432 & 2.654 & 2.076 \\
$x_{d}^{\prime}$ p.u. & 0.254 & 0.302 & 0.365 & 0.282 \\
$x_{T}$ p.u. & 0.123 & 0.115 & 0.132 & 0.142 \\
$x_{a d}$ p.u. & 1.723 & 1.723 & 1.723 & 1.723 \\
$T_{d o}^{\prime}$ p.u. & 6.45 & 7.68 & 8.24 & 7.04 \\
$M$ s & 4.0 & 5.2 & 6.4 & 4.5 \\
$D$ p.u. & 5.0 & 3.0 & 3.0 & 4.5 \\
$P_{m}$ p.u. & 1.00 & 1.10 & 1.15 & 1.05 \\
\hline \multicolumn{2}{r}{} \\
\hline
\end{tabular}

Table 2: Transformer parameters.

\begin{tabular}{llcccc}
\hline Bus & Resist. & React. & Bus & Resist. & React. \\
\hline $1-3$ & 0.0034 & 0.0200 & $7-9$ & 0.0114 & -0.0552 \\
$1-4$ & 0.0067 & 0.0350 & $8-9$ & 0.0098 & -0.0430 \\
$3-6$ & 0.0123 & -0.0450 & $8-14$ & 0.0072 & -0.0342 \\
$5-6$ & 0.0087 & -0.0320 & $9-13$ & 0.0023 & 0.0215 \\
$5-9$ & 0.1376 & -0.6543 & $10-12$ & 0.0069 & -0.0307 \\
$6-7$ & 0.1203 & -0.5120 & $11-12$ & 0.1480 & -0.6056 \\
$7-8$ & 0.0033 & -0.0160 & $9-10$ & 0.0082 & 0.0429 \\
\hline
\end{tabular}

where $i=1,2,3,4$, is the number of generator

$$
\begin{gathered}
P_{e i}(t)=E_{q i}^{\prime}(t) \sum_{j=1}^{N} E_{q j}^{\prime}\left(t-\tau_{i j}\right)\left[B_{i j} \sin \left(\delta_{i}(t)-\delta_{j}\left(t-\tau_{i j}\right)\right)+G_{i j} \cos \left(\delta_{i}(t)-\delta_{j}\left(t-\tau_{i j}\right)\right)\right], \\
I_{d i}(t)=\sum_{j=1}^{N} E_{q j}^{\prime}\left(t-\tau_{i j}\right)\left[G_{i j} \sin \left(\delta_{i}(t)-\delta_{j}\left(t-\tau_{i j}\right)\right)-B_{i j} \cos \left(\delta_{i}(t)-\delta_{j}\left(t-\tau_{i j}\right)\right)\right] .
\end{gathered}
$$

The generator parameter notions are introduced in Appendix B. The system parameter values used in the simulation are given in Tables 1 and 2.

The decentralized coordinated excitation control input limitations are

$$
-3 \leq v_{f i}=u_{l p s s}(t)+u_{s p s s}(t) \leq 6, \quad i=1,2,3,4 .
$$

The fault considered in the simulation is a symmetrical three-phase short circuit fault that occurs on the transmission line between the bus- 6 and the bus-7. $\zeta$ is the fraction of the line to the left of the fault. If $\zeta=0$, the fault is on the bus bar of no. $6, \zeta=0.5$ puts the fault in the middle of the bus- 6 and the bus- 7 . The fault sequence considered is the following.

Stage 1. The system is in prefault steady state.

Stage 2. A fault occurs at $t=0.1 \mathrm{~s}$.

Stage 3. The fault is removed by opening the breakers of the faulted line at $t=0.25 \mathrm{~s}$. 
Stage 4. The transmission lines are restored with the fault cleared at $t=1.0 \mathrm{~s}$.

Stage 5. The system is in post-fault state.

The triangle type membership functions are proposed for the fuzzy logic model with if-then rules in this paper. To minimize the design effort and complexity, we try to use as few rules as possible to represent the system. Here, considering the paper length, the coefficient matrices of the T-S fuzzy model are not displayed. For simulation study, four operating points are selected as follows.

Case 1. The operating points are

$$
\begin{gathered}
{\left[\begin{array}{l}
\delta_{10} \\
\delta_{20} \\
\delta_{30} \\
\delta_{40}
\end{array}\right]=\left[\begin{array}{l}
54.18^{\circ} \\
54.35^{\circ} \\
54.30^{\circ} \\
53.24^{\circ}
\end{array}\right]=\left[\begin{array}{l}
z_{11} \\
z_{21} \\
z_{31} \\
z_{41}
\end{array}\right],\left[\begin{array}{l}
P_{m 10} \\
P_{m 20} \\
P_{m 30} \\
p_{m 40}
\end{array}\right]=\left[\begin{array}{l}
1.10 \\
1.10 \\
1.05 \\
1.05
\end{array}\right] \text { p.u. }=\left[\begin{array}{l}
z_{12} \\
z_{22} \\
z_{32} \\
z_{42}
\end{array}\right],} \\
{\left[\begin{array}{l}
V_{t 1} \\
V_{t 2} \\
V_{t 3} \\
V_{t 4}
\end{array}\right]=\left[\begin{array}{l}
1.04 \\
1.02 \\
1.01 \\
1.02
\end{array}\right] \text { p.u. }=\left[\begin{array}{l}
z_{13} \\
z_{23} \\
z_{33} \\
z_{43}
\end{array}\right],}
\end{gathered}
$$

Case 2. The operating points are

$$
\begin{gathered}
{\left[\begin{array}{l}
\delta_{10} \\
\delta_{20} \\
\delta_{30} \\
\delta_{40}
\end{array}\right]=\left[\begin{array}{l}
32.98^{\circ} \\
33.22^{\circ} \\
31.35^{\circ} \\
35.11^{\circ}
\end{array}\right]=\left[\begin{array}{l}
z_{11} \\
z_{21} \\
z_{31} \\
z_{41}
\end{array}\right],\left[\begin{array}{l}
P_{m 10} \\
P_{m 20} \\
P_{m 30} \\
p_{m 40}
\end{array}\right]=\left[\begin{array}{l}
0.30 \\
0.40 \\
0.30 \\
0.35
\end{array}\right] \text { p.u. }=\left[\begin{array}{l}
z_{12} \\
z_{22} \\
z_{32} \\
z_{42}
\end{array}\right],} \\
{\left[\begin{array}{l}
V_{t 1} \\
V_{t 2} \\
V_{t 3} \\
V_{t 4}
\end{array}\right]=\left[\begin{array}{l}
0.83 \\
0.87 \\
0.85 \\
0.84
\end{array}\right] \text { p.u. }=\left[\begin{array}{l}
z_{13} \\
z_{23} \\
z_{33} \\
z_{43}
\end{array}\right],}
\end{gathered}
$$

Case 3. The operating points are

$$
\left[\begin{array}{l}
\delta_{10} \\
\delta_{20} \\
\delta_{30} \\
\delta_{40}
\end{array}\right]=\left[\begin{array}{l}
41.84^{\circ} \\
40.12^{\circ} \\
40.65^{\circ} \\
41.15^{\circ}
\end{array}\right]=\left[\begin{array}{l}
z_{11} \\
z_{21} \\
z_{31} \\
z_{41}
\end{array}\right], \quad\left[\begin{array}{l}
P_{m 10} \\
P_{m 20} \\
P_{m 30} \\
p_{m 40}
\end{array}\right]=\left[\begin{array}{l}
0.90 \\
0.92 \\
0.91 \\
0.90
\end{array}\right] \text { p.u. }=\left[\begin{array}{l}
z_{12} \\
z_{22} \\
z_{32} \\
z_{42}
\end{array}\right],
$$




$$
\left[\begin{array}{l}
V_{t 1} \\
V_{t 2} \\
V_{t 3} \\
V_{t 4}
\end{array}\right]=\left[\begin{array}{l}
0.94 \\
0.93 \\
0.93 \\
0.94
\end{array}\right] \text { p.u. }=\left[\begin{array}{l}
z_{13} \\
z_{23} \\
z_{33} \\
z_{43}
\end{array}\right],
$$

Case 4. The operating points are

$$
\begin{gathered}
{\left[\begin{array}{l}
\delta_{10} \\
\delta_{20} \\
\delta_{30} \\
\delta_{40}
\end{array}\right]=\left[\begin{array}{l}
71.24^{\circ} \\
72.75^{\circ} \\
73.31^{\circ} \\
71.23^{\circ}
\end{array}\right]=\left[\begin{array}{l}
z_{11} \\
z_{21} \\
z_{31} \\
z_{41}
\end{array}\right],\left[\begin{array}{l}
P_{m 10} \\
P_{m 20} \\
P_{m 30} \\
p_{m 40}
\end{array}\right]=\left[\begin{array}{l}
1.10 \\
1.00 \\
1.10 \\
1.00
\end{array}\right] \text { p.u. }=\left[\begin{array}{l}
z_{12} \\
z_{22} \\
z_{32} \\
z_{42}
\end{array}\right],} \\
{\left[\begin{array}{l}
V_{t 1} \\
V_{t 2} \\
V_{t 3} \\
V_{t 4}
\end{array}\right]=\left[\begin{array}{l}
1.01 \\
1.01 \\
1.02 \\
1.01
\end{array}\right] \text { p.u. }=\left[\begin{array}{l}
z_{13} \\
z_{23} \\
z_{33} \\
z_{43}
\end{array}\right],}
\end{gathered}
$$

Next, we will test the effectiveness of proposed controller at different fault locations.

We solve the control parameters using the LMI optimization toolbox in Matlab. When the operating points are chosen in Case 1 , we find that the system is robustly stable for any constant time delays that satisfy $0 \leq \bar{\tau} \leq 1.5643 \mathrm{~s}$. Figures 2,3 , and 4 compares the power angles of the generators no. 2 and no. 3 with different fault locations: $\zeta=0.05, \zeta=0.50$, $\zeta=0.95$.

From the results shown above, it can be shown that the proposed decentralized controller can rapidly damp down power angle oscillation of the generators no. 2 and no. 3 at different fault locations.

In Figure 5, the power angle responses of generator no. 2 are shown, respectively, for different maximum time delays with $\zeta=0.50$ in Case 2. It can be seen from Figure 5 that the proposed controller still ensures sufficient damping of system oscillation and keep the system stable regardless of variations of maximum time delay. It demonstrates that the delaydependent method is effective for all time delay whose sizes are not larger than the upper bound.

When the operating points are chose in Case 3, the power angle responses of all generators with $\zeta=1.0$ are given in Figure 6 .

It can be seen from Figure 6 that not only the generator no. 2 and no. 3 settle down quickly, but also the others have better dynamic performances by the proposed controller.

The terminal voltages of the generator no. 2 and no. 3 with fault location $\zeta=0.30$ are shown in Figure 7. From the results of Figure 7, it can be shown that the proposed control scheme guarantees better voltage quality. 


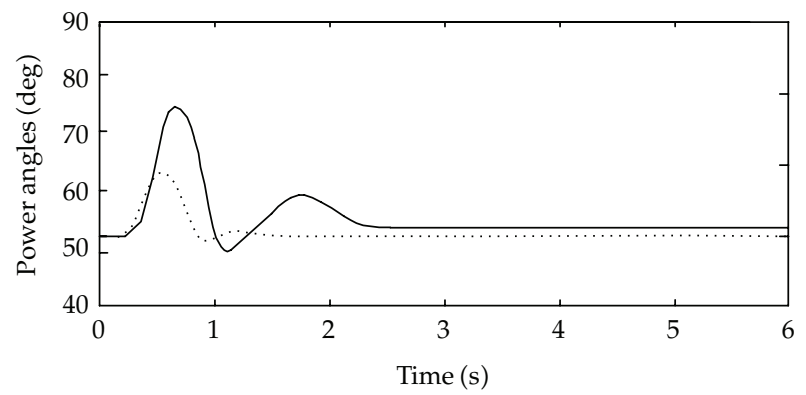

Figure 2: The responses of power angle of the generator no. 2 and no. 3 with fault location $\zeta=0.05$ (The solid line: the generator no. 2, the dotted line: the generator no. 3 ).

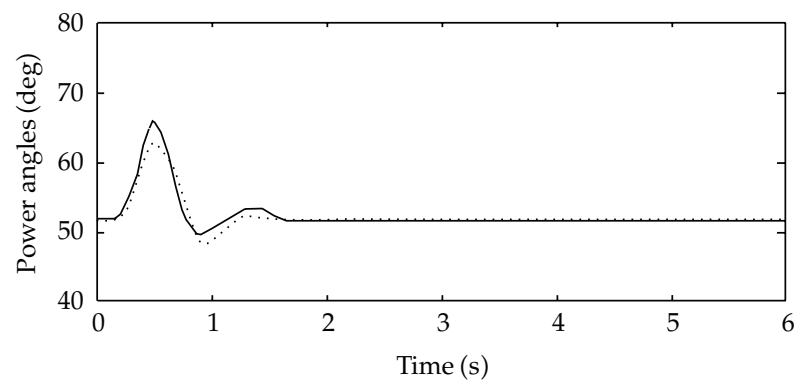

Figure 3: The responses of power angle of the generator no. 2 and no. 3 with fault location $\zeta=0.50$ (The solid line: the generator no. 2, the dotted line: the generator no. 3 ).

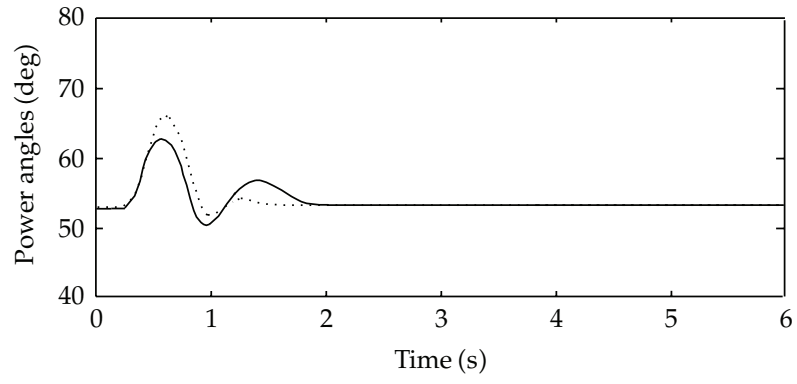

Figure 4: The responses of power angle of the generator no. 2 and no. 3 with fault location $\zeta=0.95$ (The solid line: the generator no. 2, the dotted line: the generator no. 3).

\section{Conclusion}

Based on decentralized state feedback control structure, a delay-dependent $H \infty$ robust fuzzy control method is presented for stabilization performance enhancement of the large systems that are nonlinear, interconnected, time delay, and uncertain by using T$S$ fuzzy model. The main contribution of this paper is to effectively deal with the problems related to nonlinearities, time delays and uncertainties in large systems so that the system can be stabilized for all considered uncertainties and time delays whose sizes are not larger than their upper bounds. Another contribution is that the sufficient conditions for existence of the controller are transformed into the problem of LMIs, 


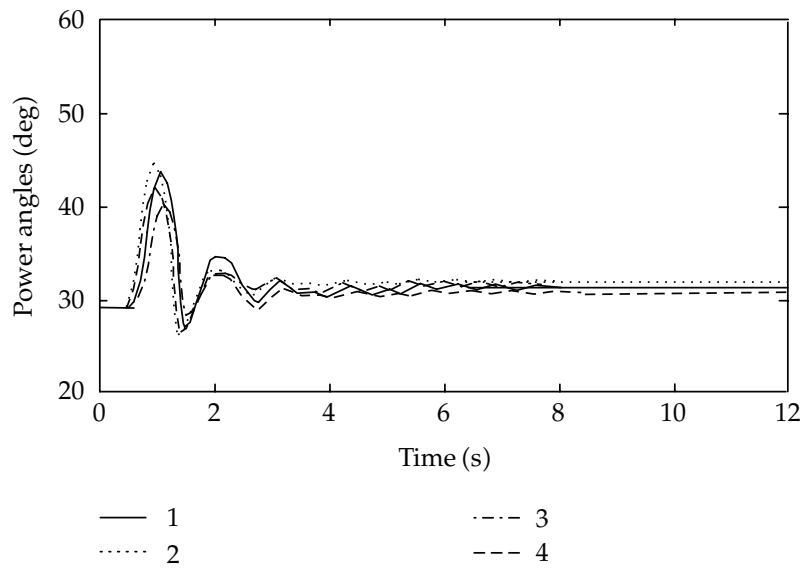

Figure 5: The responses of power angle of the generator no. 2 with fault location $\zeta=0.50$ (line no. 1 : maximum time delays are $100 \mathrm{~ms}$; line no. 2: maximum time delays are $400 \mathrm{~ms}$; line no. 3: maximum time delays are $40 \mathrm{~ms}$; line no. 4: maximum time delays are $200 \mathrm{~ms}$ ).

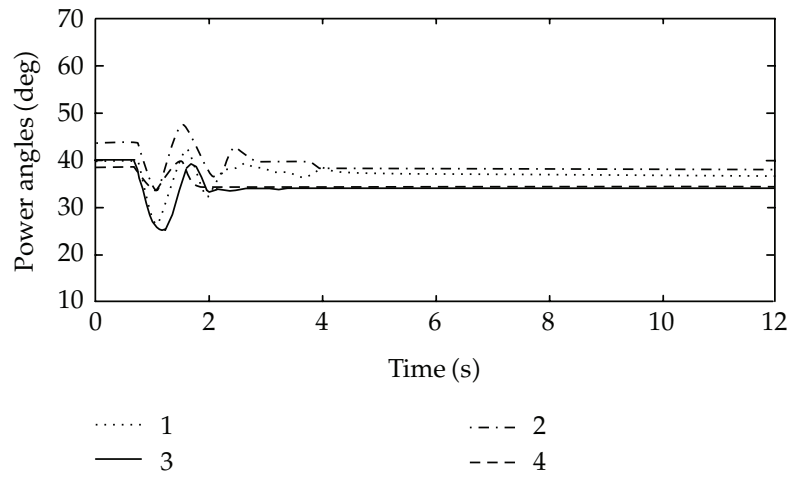

Figure 6: The power angle responses of the all generator with fault location $\zeta=1.0$ (Line no. 1: the generator no. 1 ; line no. 2: the generator no. 2; line no. 3: the generator no. 3; line no. 4: the generator no. 4).

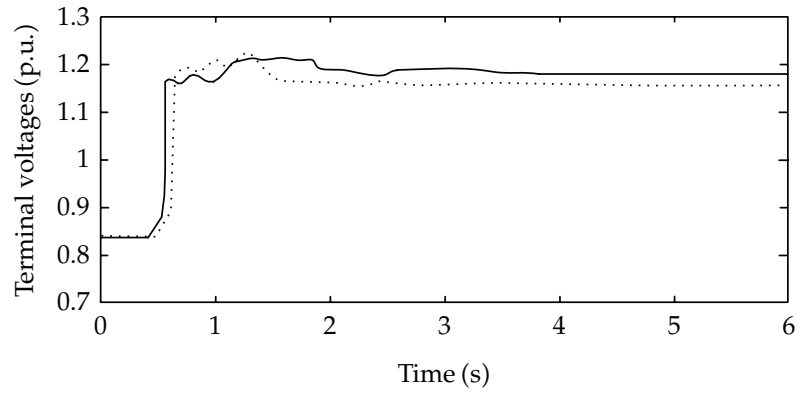

Figure 7: Terminal voltages of generators no. 2 and no. 3 with fault location $\zeta=0.30$ (The solid line: the generator no. 2, the dotted line: the generator no. 3). 
by which the $H \infty$ performance index and control parameters can be solved efficiently and rapidly by using convex optimization techniques via the available LMI toolbox. It enables controller to be more feasible and convenient for real engineering application. The proposed robust control scheme is demonstrated on a large four-machine power system. Simulation results have shown that the dynamic performance of the system is greatly improved regardless of different operating points, various time delays and faults in different locations.

\section{Appendices}

\section{A. The proof of the Theorem 3.5}

Proof. The derivative of $V_{i 1}(t)$ along the trajectory of system (3.6)

$$
\begin{aligned}
& \dot{V}_{i 1}(t)=\sum_{k=1}^{l} \sum_{m=1}^{l} h_{k}\left(z_{i}(t)\right) h_{m}\left(z_{i}(t)\right) \\
& \times\left\{2 \mathbf{x}_{i}^{T}(t) \mathbf{P}_{i i}\left[\mathbf{A}_{i k}+\mathbf{B}_{1 i k} \mathbf{K}_{i m}+\mathbf{H}_{i k} \mathbf{F}_{i k}(t)\left(\mathbf{E}_{1 i k}+\mathbf{E}_{2 i k} \mathbf{K}_{i m}\right)+\mathbf{A}_{d i k}+\mathbf{H}_{i k} \mathbf{F}_{i k}(t) \mathbf{E}_{3 i k}\right] \mathbf{x}_{i}(t)\right. \\
& -\int_{t-\tau_{i i}}^{t} 2 \mathbf{x}_{i}^{T}(t) \mathbf{P}_{i i}\left(\mathbf{A}_{d i k}+\mathbf{H}_{i k} \mathbf{F}_{i k}(t) \mathbf{E}_{3 i k}\right) \dot{\mathbf{x}}_{i}(\alpha) d \alpha \\
& +\sum_{j=1, j \neq i}^{N}\left[2 \mathbf{x}_{i}^{T}(t) \mathbf{P}_{i i}\left(\mathbf{A}_{i j k}+\mathbf{H}_{i k} \mathbf{F}_{i k}(t) \mathbf{E}_{4 i j k}\right) \mathbf{x}_{j}(t)\right. \\
& \left.+2 \mathbf{x}_{i}^{T}(t) \mathbf{P}_{i i}\left(\mathbf{A}_{d i j k}+\mathbf{H}_{i k} \mathbf{F}_{i k}(t) \mathbf{E}_{5 i j k}\right) \mathbf{x}_{j}\left(t-\tau_{i j}\right)\right] \\
& \left.+2 \mathbf{x}_{i}^{T}(t) \mathbf{P}_{i i}\left(\mathbf{B}_{2 i k}+\mathbf{H}_{i k} \mathbf{F}_{i k}(t) \mathbf{E}_{6 i k}\right) \boldsymbol{\omega}_{i}(t)\right\} \text {. }
\end{aligned}
$$

By Lemma 3.3, if $\left[\begin{array}{ll}\mathbf{X}_{i i} & \mathbf{Y}_{i i} \\ \mathbf{Y}_{i i}^{T} & \mathbf{Z}_{i i}\end{array}\right] \geq 0$, we obtains

$$
\begin{aligned}
-2 \mathbf{x}_{i}^{T}(t) & \mathbf{P}_{i i}\left(\mathbf{A}_{d i k}+\mathbf{H}_{i k} \mathbf{F}_{i k}(t) \mathbf{E}_{3 i k}\right) \dot{\mathbf{x}}_{i}(\alpha) \\
\leq & \mathbf{x}_{i}^{T}(t) \mathbf{X}_{i i} \mathbf{x}_{i}(t)+\dot{\mathbf{x}}_{i}^{T}(\alpha)\left[\mathbf{Y}_{i i}^{T}-\left(\mathbf{P}_{i i}\left(\mathbf{A}_{d i k}+\mathbf{H}_{i k} \mathbf{F}_{i k}(t) \mathbf{E}_{3 i k}\right)\right)^{T}\right] \mathbf{x}_{i}(t) \\
& +\mathbf{x}_{i}^{T}(t)\left[\mathbf{Y}_{i i}-\mathbf{P}_{i i}\left(\mathbf{A}_{d i k}+\mathbf{H}_{i k} \mathbf{F}_{i k}(t) \mathbf{E}_{3 i k}\right)\right] \dot{\mathbf{x}}_{i}(\alpha)+\dot{\mathbf{x}}_{i}^{T}(\alpha) \mathbf{Z}_{i i} \dot{\mathbf{x}}_{i}(\alpha)
\end{aligned}
$$


Then, we gain

$$
\begin{gathered}
\dot{V}_{i 1}(t) \leq \sum_{k=1}^{l} \sum_{m=1}^{l} \sum_{j=1, j \neq i}^{N} h_{k}\left(z_{i}(t)\right) h_{m}\left(z_{i}(t)\right) \\
\times\left\{\begin{array}{c}
\mathbf{x}_{i}^{T}(t)\left\{\mathbf{P}_{i i}\left[\mathbf{A}_{i k}+\mathbf{B}_{1 i k} \mathbf{K}_{i m}+\mathbf{H}_{i k} \mathbf{F}_{i k}(t)\left(\mathbf{E}_{1 i k}+\mathbf{E}_{2 i k} \mathbf{K}_{i m}\right)\right]\right. \\
\left.+\left[\mathbf{A}_{i k}+\mathbf{B}_{1 i k} \mathbf{K}_{i m}+\mathbf{H}_{i k} \mathbf{F}_{i k}(t)\left(\mathbf{E}_{1 i k}+\mathbf{E}_{2 i k} \mathbf{K}_{i m}\right)\right]^{T} \mathbf{P}_{i i}+\bar{\tau} \mathbf{X}_{i i}+\mathbf{Y}_{i i}+\mathbf{Y}_{i i}^{T}\right\} \mathbf{x}_{i}(t)
\end{array}\right. \\
-2 \mathbf{x}_{i}^{T}(t)\left[\mathbf{Y}_{i i}-\mathbf{P}_{i i}\left(\mathbf{A}_{d i k}+\mathbf{H}_{i k} \mathbf{F}_{i k}(t) \mathbf{E}_{3 i k}\right)\right] \mathbf{x}_{i}\left(t-\tau_{i i}\right)+\int_{t-\tau_{i i}}^{t} \dot{\mathbf{x}}_{i}^{T}(\alpha) \mathbf{Z}_{i i} \dot{\mathbf{x}}_{i}(\alpha) d \alpha \\
+\sum_{j=1, j \neq i}^{N}\left[2 \mathbf{x}_{i}^{T}(t) \mathbf{P}_{i i}\left(\mathbf{A}_{i j k}+\mathbf{H}_{i k} \mathbf{F}_{i k}(t) \mathbf{E}_{4 i j k}\right) \mathbf{x}_{j}(t)\right. \\
\left.+2 \mathbf{x}_{i}^{T}(t) \mathbf{P}_{i i}\left(\mathbf{A}_{d i j k}+\mathbf{H}_{i k} \mathbf{F}_{i k}(t) \mathbf{E}_{5 i j k}\right) \mathbf{x}_{j}\left(t-\tau_{i j}\right)\right] \\
\left.+2 \mathbf{x}_{i}^{T}(t) \mathbf{P}_{i i}\left(\mathbf{B}_{2 i k}+\mathbf{H}_{i k} \mathbf{F}_{i k}(t) \mathbf{E}_{6 i k}\right) \boldsymbol{\omega}_{i}(t)\right\}, \\
\dot{V}_{i 2}(t)=\mathbf{x}_{i}^{T}(t) \mathbf{S}_{i i} \mathbf{x}_{i}(t)-\mathbf{x}_{i}^{T}\left(t-\tau_{i i}\right) \mathbf{S}_{i i} \mathbf{x}_{i}\left(t-\tau_{i i}\right) \\
\dot{V}_{i 3}(t)=\tau_{i i} \dot{\mathbf{x}}_{i}^{T}(t) \mathbf{Z}_{i i} \dot{\mathbf{x}}_{i}(t)-\int_{t-\tau_{i i}}^{t} \dot{\mathbf{x}}_{i}^{T}(\alpha) \mathbf{Z}_{i i} \dot{\mathbf{x}}_{i}(\alpha) d \alpha .
\end{gathered}
$$

Based on the delay-independent Lyapunov function and the $H \infty$ performance (3.9), it is easy to obtain:

$$
\begin{aligned}
& \int_{0}^{t_{f}} \mathbf{y}_{i}^{T} \mathbf{y}_{i} d t \\
& =V_{i}(0)-V_{i}\left(t_{f}\right)+\int_{0}^{t_{f}}\left[\mathbf{y}_{i}^{T} \mathbf{y}_{i}+\dot{V}_{i}(t)\right] d t \\
& \leq V_{i}(0)+\int_{0}^{t_{f}} \sum_{k=1}^{l} \sum_{m=1}^{l} \sum_{j=1, j \neq i}^{N} h_{k}\left(z_{i}(t)\right) h_{m}\left(z_{i}(t)\right) \\
& \quad \times\left\{\mathbf { x } _ { i } ^ { T } ( t ) \left\{\begin{array}{l}
\mathbf{P}_{i i}\left[\mathbf{A}_{i k}+\mathbf{B}_{1 i k} \mathbf{K}_{i m}+\mathbf{H}_{i k} \mathbf{F}_{i k}(t)\left(\mathbf{E}_{1 i k}+\mathbf{E}_{2 i k} \mathbf{K}_{i m}\right)\right] \\
\left.\quad+\left[\mathbf{A}_{i k}+\mathbf{B}_{1 i k} \mathbf{K}_{i m}+\mathbf{H}_{i k} \mathbf{F}_{i k}(t)\left(\mathbf{E}_{1 i k}+\mathbf{E}_{2 i k} \mathbf{K}_{i m}\right)\right]^{T} \mathbf{P}_{i i}+\bar{\tau} \mathbf{X}_{i i}+\mathbf{Y}_{i i}+\mathbf{Y}_{i i}^{T}\right\} \mathbf{x}_{i}(t) \\
\quad+\int_{t-\tau_{i i}}^{t} \dot{\mathbf{x}}_{i}^{T}(\alpha) \mathbf{Z}_{i i} \dot{\mathbf{x}}_{i}(\alpha) d \alpha-2 \mathbf{x}_{i}^{T}(t)\left[\mathbf{Y}_{i i}-\mathbf{P}_{i i}\left(\mathbf{A}_{d i k}+\mathbf{H}_{i k} \mathbf{F}_{i k}(t) \mathbf{E}_{3 i k}\right)\right] \mathbf{x}_{i}\left(t-\tau_{i i}\right)
\end{array}\right.\right.
\end{aligned}
$$




$$
\begin{gathered}
+\sum_{j=1, j \neq i}^{N}\left[2 \mathbf{x}_{i}^{T}(t) \mathbf{P}_{i i}\left(\mathbf{A}_{i j k}+\mathbf{H}_{i k} \mathbf{F}_{i k}(t) \mathbf{E}_{4 i j k}\right) \mathbf{x}_{j}(t)\right. \\
\left.+2 \mathbf{x}_{i}^{T}(t) \mathbf{P}_{i i}\left(\mathbf{A}_{d i j k}+\mathbf{H}_{i k} \mathbf{F}_{i k}(t) \mathbf{E}_{5 i j k}\right) \mathbf{x}_{j}\left(t-\tau_{i j}\right)\right] \\
\left.+2 \mathbf{x}_{i}^{T}(t) \mathbf{P}_{i i}\left(\mathbf{B}_{2 i k}+\mathbf{H}_{i k} \mathbf{F}_{i k}(t) \mathbf{E}_{6 i k}\right) \boldsymbol{\omega}_{i}(t)\right\} \\
+\mathbf{x}_{i}^{T}(t) \mathbf{S}_{i i} \mathbf{x}_{i}(t)-\mathbf{x}_{i}^{T}\left(t-\tau_{i i}\right) \mathbf{S}_{i i} \mathbf{x}_{i}\left(t-\tau_{i i}\right)+\bar{\tau}_{i}^{T}(t) \mathbf{Z}_{i i} \dot{\mathbf{x}}_{i}(t)-\int_{t-\tau_{i i}}^{t} \dot{\mathbf{x}}_{i}^{T}(\alpha) \mathbf{Z}_{i i} \dot{\mathbf{x}}_{i}(\alpha) d \alpha \\
+\left[\left(\mathbf{C}_{i k}+\mathbf{H}_{i k} \mathbf{F}_{i k}(t) \mathbf{E}_{7 i k}\right) \mathbf{x}_{i}(t)+\left(\mathbf{D}_{i k}+\mathbf{H}_{i k} \mathbf{F}_{i k}(t) \mathbf{E}_{8 i k}\right) \boldsymbol{\omega}_{i}(t)\right]^{T} \\
\times\left[\left(\mathbf{C}_{i k}+\mathbf{H}_{i k} \mathbf{F}_{i k}(t) \mathbf{E}_{7 i k}\right) \mathbf{x}_{i}(t)+\left(\mathbf{D}_{i k}+\mathbf{H}_{i k} \mathbf{F}_{i k}(t) \mathbf{E}_{8 i k}\right) \boldsymbol{\omega}_{i}(t)\right] \\
\left.+\rho_{i}^{2} \boldsymbol{\omega}_{i}^{T}(t) \boldsymbol{\omega}_{i}(t)-\rho_{i}^{2} \boldsymbol{\omega}_{i}^{T}(t) \boldsymbol{\omega}_{i}(t)\right\} d t .
\end{gathered}
$$

By the above result and Schur [18] complement, it is easy to obtain that if (3.13) is satisfied, $\int_{0}^{t_{f}} \mathbf{y}_{i}^{T}(t) \mathbf{y}_{i}(t) d t \leq \rho_{i}^{2} \int_{0}^{t_{f}} \boldsymbol{\omega}_{i}^{T}(t) \boldsymbol{\omega}_{i}(t) d t+V_{i}(0)$. Therefore, only if (3.13) is satisfied, the system (3.6) is asymptotically stable via the decentralized fuzzy controller in (3.2) and satisfies the $H \infty$ performance in (3.9) with a prescribed $\rho_{i}^{2}$. This completes the proof.

\section{B. Notation}

$\delta_{i}(t)$ : Power angle of the $i$ th generator, in rad

$\omega_{i}(t)$ : The angular velocity of the $i$ th generator, in $\mathrm{rad} / \mathrm{s}$

$\omega_{0}$ : Synchronous machine speed, in $\mathrm{rad} / \mathrm{s}$

$E_{q i}^{\prime}(t): q$ axis transient potential, in p.u.

$V_{f i}(t)$ : Control input, excitation voltage, in p.u.

$x_{d i}: d$-axis synchronous reactance, in p.u.

$x_{d i}^{\prime}: d$-axis transient reactance, in p.u.

$Y_{i j}=G_{i j}+j B_{i j}$ : Modulus of the transfer admittance between the $i$ th and $j$ th generator, in p. u.

$P_{\mathrm{mi}}$ : Mechanical input power, in p. u., which is a constant

$P_{\mathrm{ei}}$ : Electrical power, in p. u.

$D_{i}$ : Damping coefficient of $i$ th generator

$M_{i}$ : Stored energy at rated speed, inertia constant, in seconds

$T_{d o i}^{\prime}: d$-axis open circuit transient time constant, in seconds

$I_{\mathrm{di}}$ : Direct axis current, in p.u. 


\section{Acknowledgment}

This work is supported by the National Science Foundation of China under Grants nos. 60874023 and 60974078.

\section{References}

[1] M. Jamshidi, Large-Scale System: Modeling and Control, Elsevier, New York, NY, USA, 1983.

[2] W. S. Chan and C. A. Desoer, "Eigenvalue assignment and stabilization of interconnected systems using local feedbacks," IEEE Transactions on Automatic Control, vol. 25, pp. 106-107, 1980.

[3] Y.-Y. Cao and P. M. Frank, "Stability analysis and synthesis of nonlinear time-delay systems via linear Takagi-Sugeno fuzzy models," Fuzzy Sets and Systems, vol. 124, no. 2, pp. 213-229, 2001.

[4] X. P. Guan and C. L. Chen, "Delay-dependent guaranteed cost control for T-S fuzzy systems with time delays," IEEE Transactions on Fuzzy Systems, vol. 12, no. 2, pp. 236-249, 2004.

[5] X. Liu and H. Zhang, "Delay-dependent robust stability of uncertain fuzzy large-scale systems with time-varying delays," Automatica, vol. 44, no. 1, pp. 193-198, 2008.

[6] H. Zhang and J. Yu, "LMI-based stability analysis of fuzzy large-scale systems with time delays," Chaos, Solitons \& Fractals, vol. 25, no. 5, pp. 1193-1207, 2005.

[7] R.-J. Wang, "Nonlinear decentralized state feedback controller for uncertain fuzzy time-delay interconnected systems," Fuzzy Sets and Systems, vol. 151, no. 1, pp. 191-204, 2005.

[8] C. X. Dou, Q. Q. Jia, S. J. Jin, and Z. Q. Bo, “Delay-independent decentralized stabilizer design for large interconnected power systems based on WAMS," International Journal of Electrical Power E Energy Systems, vol. 29, pp. 775-782, 2007.

[9] W. Z. Qiu, V. Vittal, and M. Khammash, “Decentralized power system stabilizer design using linear parameter varying approach," IEEE Transactions on Power Systems, vol. 19, pp. 1951-1959, 2004.

[10] S. C. Tong, S. Tong, and Q. L. Zhang, "Robust stabilization of nonlinear time-delay interconnected systems via decentralized fuzzy Control," International Journal of Innovative Computing Information and Control, vol. 4, pp. 1567-1582, 2008.

[11] O. M. Kown and J. H. Park, "Decentralized guaranteed cost control for uncertain large-scale systems using delayed feedback: LMI optimization approach," Journal of Optimization Theory and Applications, vol. 129 , no. 3, pp. 391-414, 2006.

[12] C. S. Tseng and B. S. Chen, "Fuzzy tracking control design for nonlinear dynamic systems via T-S fuzzy model," The IEEE Transactions on Fuzzy Systems, vol. 9, pp. 381-392, 2001.

[13] E. Kim and S. Kim, "Stability analysis and synthesis for an affine fuzzy control system via LNI and ILMI: continuous case," The IEEE Transactions on Fuzzy Systems, vol. 10, pp. 391-400, 2001.

[14] E. Kim and H. Lee, "New approaches to related quadratic stability condition of fuzzy control systems," The IEEE Transactions on Fuzzy Systems, vol. 8, pp. 523-534, 2000.

[15] J. W. Cai, S. S. Hu, and H. F. Tao, "Fuzzy static output-feedback guaranteed cost reliable control for uncertain nonlinear systems with time-delay," International Journal of Innovative Computing Information and Control, vol. 4, pp. 3409-3420, 2008.

[16] Y. S. Moon, P. Park, W. H. Kwon, and Y. S. Lee, "Delay-dependent robust stabilization of uncertain state-delayed systems," International Journal of Control, vol. 74, no. 14, pp. 1447-1455, 2001.

[17] I. R. Petersen and C. V. Hollot, "A Riccati equation approach to the stabilization of uncertain linear systems," Automatica, vol. 22, no. 4, pp. 397-411, 1986

[18] S. Boyd, L. El Ghaoui, E. Feron, and V. Balakrishnan, Linear Matrix Inequalities in System and Control Theory, vol. 15 of SIAM Studies in Applied Mathematics, Society for Industrial and Applied Mathematics (SIAM), Philadelphia, Pa, USA, 1994.

[19] N. Yadaiah and R. N. Venkata, "Linearisation of multi-machine power system: modeling and controlA survey," International Journal of Electrical Power E Energy Systems, vol. 29, pp. 297-311, 2007. 


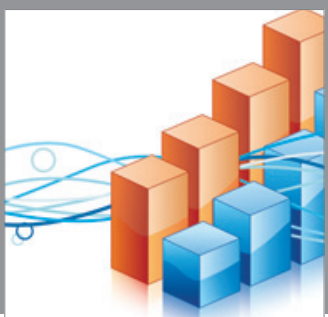

Advances in

Operations Research

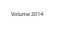

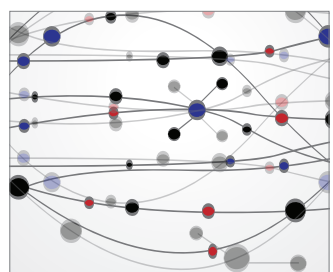

\section{The Scientific} World Journal
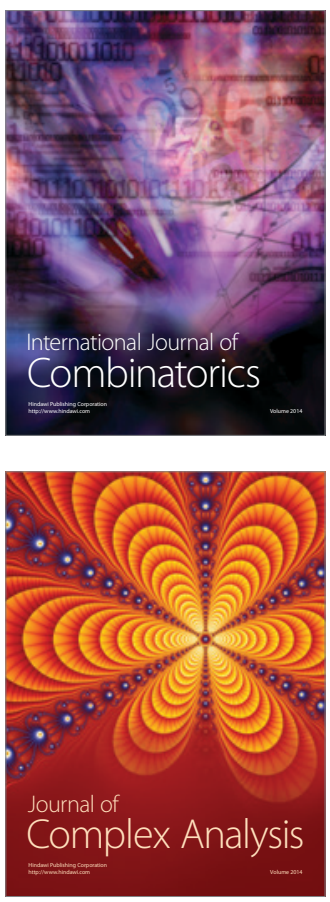

International Journal of

Mathematics and

Mathematical

Sciences
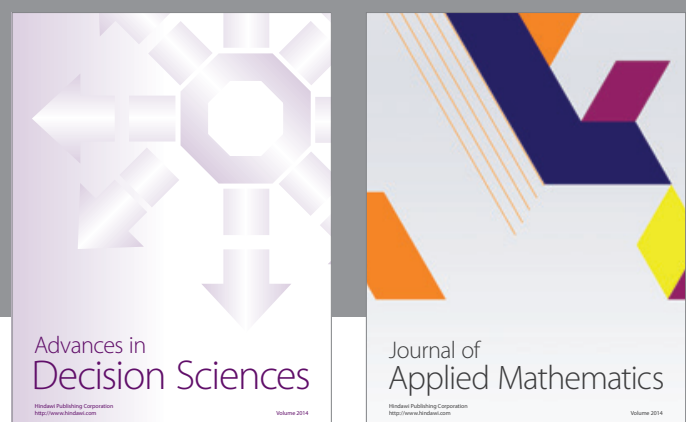

Journal of

Applied Mathematics
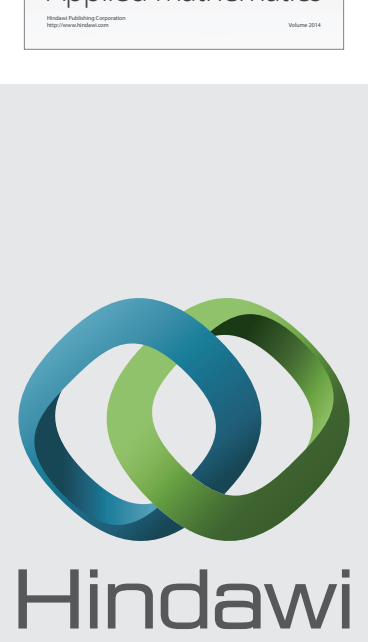

Submit your manuscripts at http://www.hindawi.com
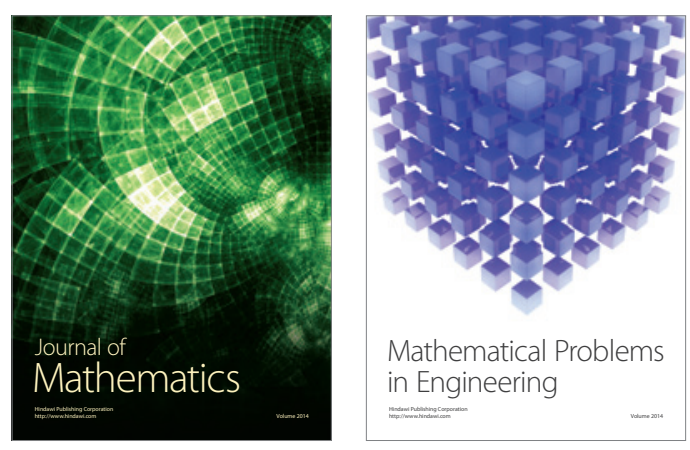

Mathematical Problems in Engineering
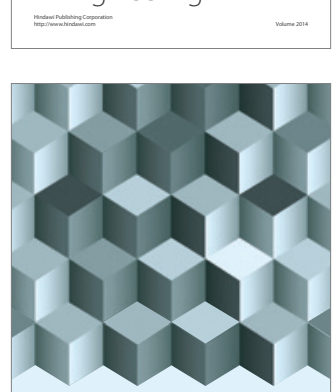

Journal of

Function Spaces
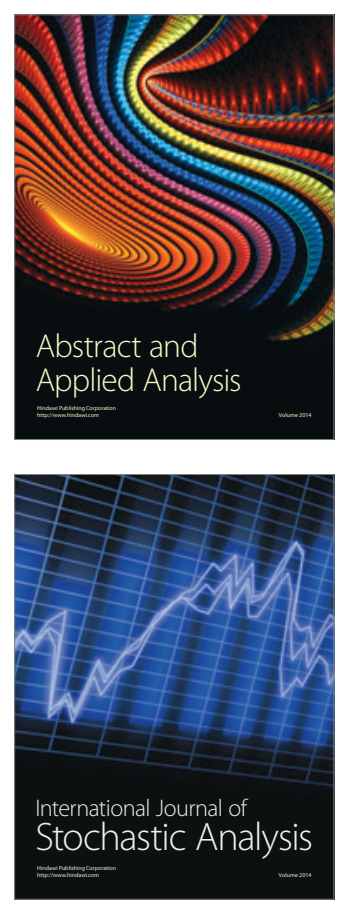

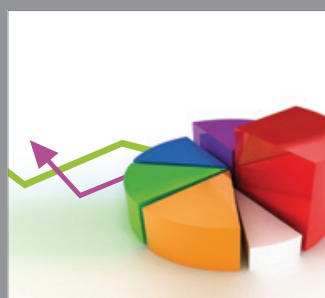

ournal of

Probability and Statistics

Promensencen
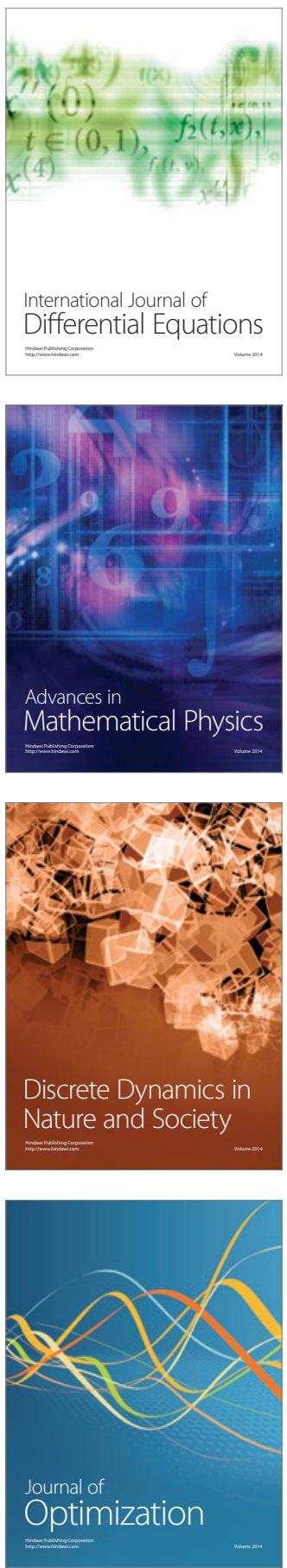